\title{
Determination of Planetary Boundary Layer Height on Short Spatial and Temporal Scales: A Demonstration of the Covariance Wavelet Transform in Ground-Based Wind Profiler and Lidar Measurements*
}

\author{
JAIME C. COMPTON \\ Physics Department, University of Maryland, Baltimore County, Baltimore, Maryland \\ Ruben Delgado And Timothy A. BerkofF \\ Joint Center for Earth Systems Technology, University of Maryland, Baltimore County, Baltimore, Maryland \\ RAYMOND M. HOFF \\ Physics Department, and Joint Center for Earth Systems Technology, University of Maryland, \\ Baltimore County, Baltimore, Maryland
}

(Manuscript received 30 May 2012, in final form 20 February 2013)

\begin{abstract}
This article explores the application of the covariance wavelet transform (CWT) to lidar and, for the first time to the authors' knowledge, wind profiler data to examine the possibility of accurate and continuous planetary boundary layer (PBL) height measurements on short temporal resolution (1- and 15-min averages, respectively). Determining the mixing in the PBL was one goal of a study of the spatial and diurnal variations of the PBL height over Maryland for July 2011, during NASA's Earth Venture mission DISCOVER-AQ. The PBL heights derived from ground-based lidars [at University of Maryland, Baltimore County (UMBC); $39.25^{\circ} \mathrm{N}, 76.70^{\circ} \mathrm{W}$ ], a $915-\mathrm{MHz}$ wind profiler, and radiosondes (at Beltsville, Maryland; $38.92^{\circ} \mathrm{N}, 77.02^{\circ} \mathrm{W}$ ) were compared. Results from the comparison show an $R^{2}=0.89,0.92$, and 0.94 correlation between the radiosonde PBL heights and two lidars and wind profiler PBL heights, respectively. Accurate determination of the PBL height by applying the CWT to lidar and wind profilers will allow for improved air quality forecasting and understanding of regional pollution dynamics.
\end{abstract}

\section{Introduction}

The earth's lowest atmosphere is demarked by a planetary boundary layer (PBL), which is the lowest layer of the atmosphere in contact with the surface. The PBL is defined by the exchange of heat, moisture, and momentum with the surface. Daytime PBL heights are primarily limited by surface heating and buoyancy of upward rising air parcels, and constrained by the

\footnotetext{
* Supplemental information related to this paper is available at the Journals Online website: http://dx.doi.org/10.1175/JTECH-D12-00116.s1.

Corresponding author address: Raymond M. Hoff, University of Maryland, Baltimore County, 1000 Hilltop Circle, Baltimore, MD 21250 .

E-mail: hoff@umbc.edu
}

temperature lapse rate of the overlying air. Above the PBL are other thermodynamically defined boundaries, such as residual layers from prior days and advection (Stull 1988), and ultimately in the transition to the stratosphere at the tropopause. Aerosols are primarily created and trapped within the PBL, but they may also be constrained by more elevated inversions if they have been lofted above the PBL by heating. The diurnal cycle of the PBL is discussed by Stull (1988). Conceptually, during the day, the PBL is composed of a lower layer known as the surface layer (SL) of a few tens of meters, a middle layer known as the mixed layer (ML) of depth up to a few kilometers, and capped by a top layer known as the entrainment zone (EZ) of depth of a few hundred meters. During the night, the ML collapses into the nocturnal boundary layer (NBL) that is at most a few hundred meters thick. Above the NBL is the residual layer (RL), left over from the 
previous day's ML. The RL is enclosed by the capping inversion $(\mathrm{CI})$.

Atmospheric variables such as potential temperature, aerosol concentration, and specific humidity usually experience sharp gradients at the top of the PBL. This interface is not generally a hard lid, and the EZ is a region of a few hundred meters where the exchange of air between the PBL and free troposphere occurs at scales of a few hundred meters horizontally. Concentrations of aerosols and moisture within the PBL are entrained and mixed into the cleaner and drier free troposphere (Cohn and Angevine 2000) and some downward flux of clear air also occurs. Radiosondes are used to measure temperature and specific humidity throughout the atmosphere, and can detect the layer in which the potential temperature capping inversion and specific humidity gradients occurs in the EZ. A lidar measures the intensity of backscattered light off particles and gases in the atmosphere as a function of distance, and elastic backscattering lidars are used to study the vertical aerosol profile of the atmosphere. The contrast in aerosol concentrations in the mixed layer relative to the cleaner free troposphere allows for the planetary boundary layer height (PBLH) to be detected by lidar.

In this study, we also employ the $915-\mathrm{MHz}$ wind profiler radar reflectivity and signal-to-noise ratio (SNR) data that are proportional to the refractive index structure constant $C_{n}^{2}$ (Ottersten 1969). This is highly dependent on the gradient in specific humidity (Cohn and Angevine 2000). The gradient in specific humidity caused by the contrast in concentrations in the PBL relative to the drier free troposphere allows for detection of the wind profiler PBLH.

Even with these three instruments available, there exists no sufficient national coverage to address the challenge of determining the height of the PBL throughout its diurnal cycle. The National Research Council (NRC 2009) has recommended a "network of networks" that builds new and integrates already existing radiosonde launch sites, wind profilers, and lidars into a national network to address the current inadequacies in determining the PBL height. They have stated that after 60 years of remote sensing research, it is astounding that the PBL, an important meteorological variable, is not measured regularly throughout its diurnal cycle (NRC 2009).

Because of the harmful effects on health caused by particulate matter and ozone (Lippmann 1991; Bell et al. 2004; WHO 2012), accurate forecasting of air quality conditions is needed for public well-being. The PBLH is one of the diagnostics for uncertainties in the models (Angevine et al. 1994). The PBL is important because it contains most of the aerosols, and its height determines the volume available for aerosol dispersion (Baars et al. 2008). Models with inaccurate PBLH generally will not predict surface concentrations correctly and are likely to be compensated by tuning of other parameters, such as chemistry and emissions, in order to adjust surface concentrations to reality. Because of this, tools and methods that can accurately determine the height of the PBL are needed. Especially important are methods that could be assimilated in real time into these models. This study will help determine the bestsuited instrumentation and methodology that will satisfy the spatial and temporal requirements necessary to monitor daytime PBL heights. Experimental platforms evaluated in this study, radiosondes, lidars, and the wind profiler are described in section 2. Section 3 describes the methods of determining the PBLH from the lidar and wind profiler data in this study. Section 4 discusses the comparisons between PBLHs from the radiosonde launches and those determined from lidars and the wind profiler.

\section{Experimental setup}

Deriving Information on Surface Conditions from Column and Vertically Resolved Observation Relevant to Air Quality (DISCOVER-AQ) is a National Aeronautics and Space Administration (NASA) Earth Venture mission designed to examine the representativeness of column optical properties from satellites to groundbased air quality. The first campaign was carried out in the Baltimore-Washington, D.C., metropolitan area during July 2011.

Normal launch times of National Weather Service radiosondes in the eastern United States are at 0700 and 1900 local standard time, which are not well suited for detection of the convective PBL during the day. Since the DISCOVER-AQ experiment was a satellite validation and air quality mission, operations took place during daylight hours on days that were either clear or only partly cloudy. Ground-based validation data were centered on flight hours and gave valuable correlative measurements that we could use for this study. A total of 25 Vaisala RS-92 radiosondes were launched from the Howard University Beltsville Research Campus (HUBRC) in Beltsville, Maryland $\left(38.05^{\circ} \mathrm{N}, 76.88^{\circ} \mathrm{W}\right)$, in support of DISCOVER-AQ. Many of the radiosondes were flown in tandem with Vaisala ozonesondes in DISCOVER-AQ. While the ozonesonde data provided additional confirmation of PBL heights, the gradient in ozone concentration at the top of the PBL is a poorer indicator of the PBL than was the potential temperature profile. In some cases ozone was elevated above the PBL and depleted below and some cases, the reverse occurred. We chose 
not to employ ozonesonde data for this study. Vaisala RS-92 radiosonde profiles of temperature and relative humidity were used to derive the potential temperature and specific humidity profiles. The convective parcel method of determining the PBLH (Seibert et al. 2000) was used, by examining both potential temperature and humidity gradients. This was a subjective rather than objective technique.

Lidar observations were made to monitor the evolution of the PBL using the Elastic Lidar Facility (ELF), located at the University of Maryland, Baltimore County (UMBC) $\left(39.25^{\circ} \mathrm{N}, 76.70^{\circ} \mathrm{W}\right)$. The ELF lidar transmitter consists of a Continuum Surelight 532/ $1064 \mathrm{~nm} \mathrm{Nd:YAG} \mathrm{laser} \mathrm{that} \mathrm{operates} \mathrm{at} \mathrm{a} 10-\mathrm{Hz}$ repetition rate and average pulse energy of $250 \mathrm{~mJ}$ at $532 \mathrm{~nm}$. The receiver system consists of a $0.4-\mathrm{m}$ Celestron Schmidt-Cassegrain telescope that collects the backscattered light. The output from the telescope is focused to a Hamamatsu H6780 photomultiplier tube (PMT) with a 532-nm spike filter. Neutral density filters, in the optical path to the detector, are used to avoid signal saturation of the detector, since Mie scattering has such a large backscattering cross section. The filters can be replaced with higher or lower values in order to obtain the best signal under the changing atmospheric conditions. The backscattered laser signal is digitized with a Licel TR $20-160$ photon counter at $20 \mathrm{MHz}$ and averaged for 1-min temporal resolution. The resulting vertical resolution of ELF is 7.5 m. Detailed ELF system specifications and data analysis algorithms have been reported elsewhere (Comer 2003; Engel-Cox et al. 2006).

Elastic lidar observations at HUBRC were performed using a Sigma Space mini-micropulse lidar (MPL). The MPL transmitter consists of a 532-nm frequencydoubled Nd:YAG that operates at a $5000-\mathrm{Hz}$ repetition rate and average pulse energy of 3-4 $\mu \mathrm{J}$. The receiver consists of an 80-mm telescope that collects the copolarized backscattered light. The output from the telescope is conveyed to a photon-counting silicon avalanche photodiode (APD) manufactured by Perkin Elmer. The APD output is recorded by a field programmable gate array (FPGA) data system that enables display and storage of range-dependent averaged count rates on a laptop computer. The raw data are converted to aerosol attenuated backscatter by taking into account instrumental factors that include corrections for detector dead time, geometrical overlap, background subtraction, and rangesquared normalization. Recorded lidar profiles have temporal and vertical resolution of $1 \mathrm{~min}$ and $30 \mathrm{~m}$, respectively.

Wind profiler measurements were provided by the Maryland Department of the Environment's Detect,
Inc. Raptor DBS-BL LAP-3000 Upgrade radar wind profiler located at HUBRC. The profiler operates at a frequency of $915 \mathrm{MHz}$ and measures wind speed and direction by transmitting radio pulses into the atmosphere and measuring the strength and frequency shift of backscattered energy. The vertical measurements of wind speed and direction extend from approximately 100 to $4000 \mathrm{~m}$, with a resolution of $100 \mathrm{~m}$. The first range bin in the profiler can be affected by beam pattern and local ground clutter. The Beltsville profiler has not been characterized for possible effects of clutter interfering with the registration of the first digitized bin. This will be noted again below, and we estimate that the error in height registration can be as large as one bin $(100 \mathrm{~m})$. The profiler operates continuously and displays wind speed and wind direction in 15-min intervals.

\section{PBL retrieval methods}

The PBL is capped by a temperature inversion that occurs at the EZ that traps aerosols and moisture below. Above the temperature inversion, there are generally fewer aerosols and drier air. Radiosondes are used to measure the height of the potential temperature capping inversion. It has been shown that the vertical lidar backscatter and wind profiler SNR gradient is related to this temperature inversion (Melfi et al. 1985; Cohn and Angevine 2000). Figure 1 shows the potential temperature and specific humidity profiles from a radiosonde launch at 1448 UTC 16 July 2011. Figure 1 also shows ELF and MPL attenuated backscatter, and wind profiler SNR profiles. The plots of these profiles show the potential temperature capping inversion, a decrease in specific humidity, and gradients in lidar backscatter and wind profiler SNR all around $1.40 \mathrm{~km}$. An objective method for determining the height of the PBL is needed that looks for and detects these sharp gradients in lidar and wind profiler profiles. While several gradient methods have been used over the years to determine the height of the PBL using lidar (Melfi et al. 1985; Steyn et al. 1999) and wind profiler data (Angevine et al. 1994), the method that will be used here is the covariance wavelet transform (CWT). The CWT has been demonstrated to work on lidar backscatter profiles (Cohn and Angevine 2000; Davis et al. 2000; Brooks 2003) but has yet to be demonstrated to work with wind profiler SNR profiles.

The CWT is used to detect step changes in signals that contain sharp transitions (Gamage and Hagelberg 1993). The CWT utilizes a compound step function, the Haar wavelet function, defined as 

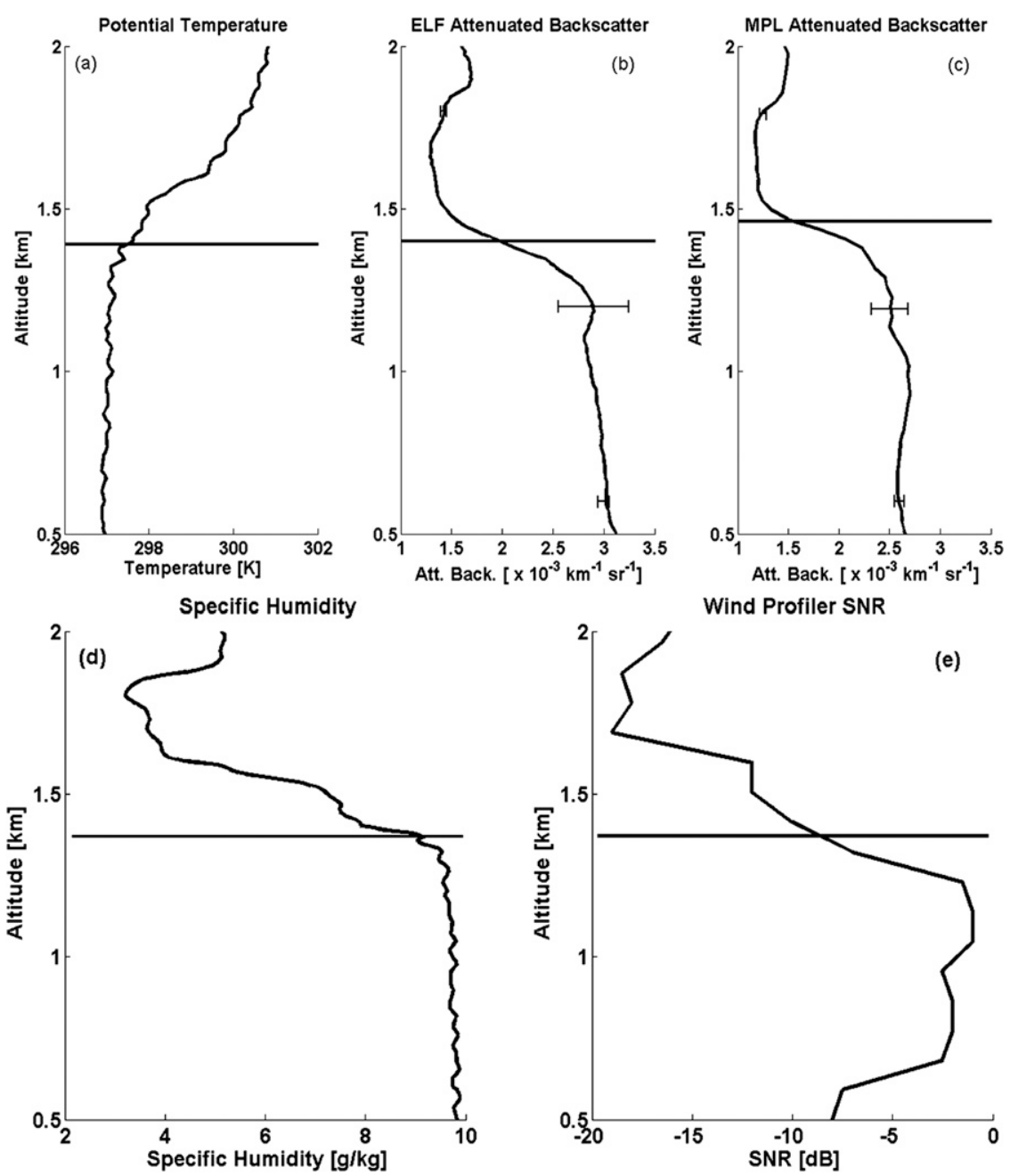

FIG. 1. Profiles of (a) potential temperature, and (b) ELF and (c) MPL attenuated backscatter at 1452 UTC 16 Jul 2011 show that the gradient in aerosol backscatter occurs within $70 \mathrm{~m}$ of the potential temperature capping inversion. Profiles of (d) specific humidity and (e) wind profiler SNR for the same time show the gradient in SNR signal occurs within $150 \mathrm{~m}$ of the height that the specific humidity begins to sharply decrease. Comparing (a)-(e), the gradients all occur around $1.40 \mathrm{~km}$ and the horizontal line is the final algorithm retrieved PBLH discussed below.

$$
h\left(\frac{z-b}{a}\right)=\left\{\begin{array}{l}
-1: b-\frac{a}{2} \leq z<b \\
+1: b \leq z \leq b+\frac{a}{2} \\
0: \text { elsewhere }
\end{array}\right\}
$$

where $h$ is the vertical distance or altitude in this application, $a$ is the spatial extent or dilation of the function, and $b$ is the center of the Haar function (Brooks 2003). The CWT of the Haar function is defined by Gamage and Hagelberg (1993) using the equation

$$
W_{f}(a, b)=a^{-1} \int_{z_{b}}^{z_{t}} f(z) h\left(\frac{z-b}{a}\right) d z
$$

where $z_{t}$ and $z_{b}$ are the top and bottom altitudes in the profile, $f(z)$ is the profile as a function of altitude, and the normalization factor $a^{-1}$ is the inverse of the dilation factor. The first step in the PBLH algorithm (Fig. 2) defines the initial conditions: the dilation and center of the Haar function values. The second step is to apply the CWT to the profile for the appropriate dilation and center of the Haar function values. The sharp gradients in the profile that are of interest are identified by local minima in the resulting wavelet covariance profile. The appropriate local minimum (discussed below) is selected as the PBLH. This process is repeated for each profile in the dataset.

Defining the initial conditions for this algorithm is crucial for it to produce correct PBLHs. The center of 


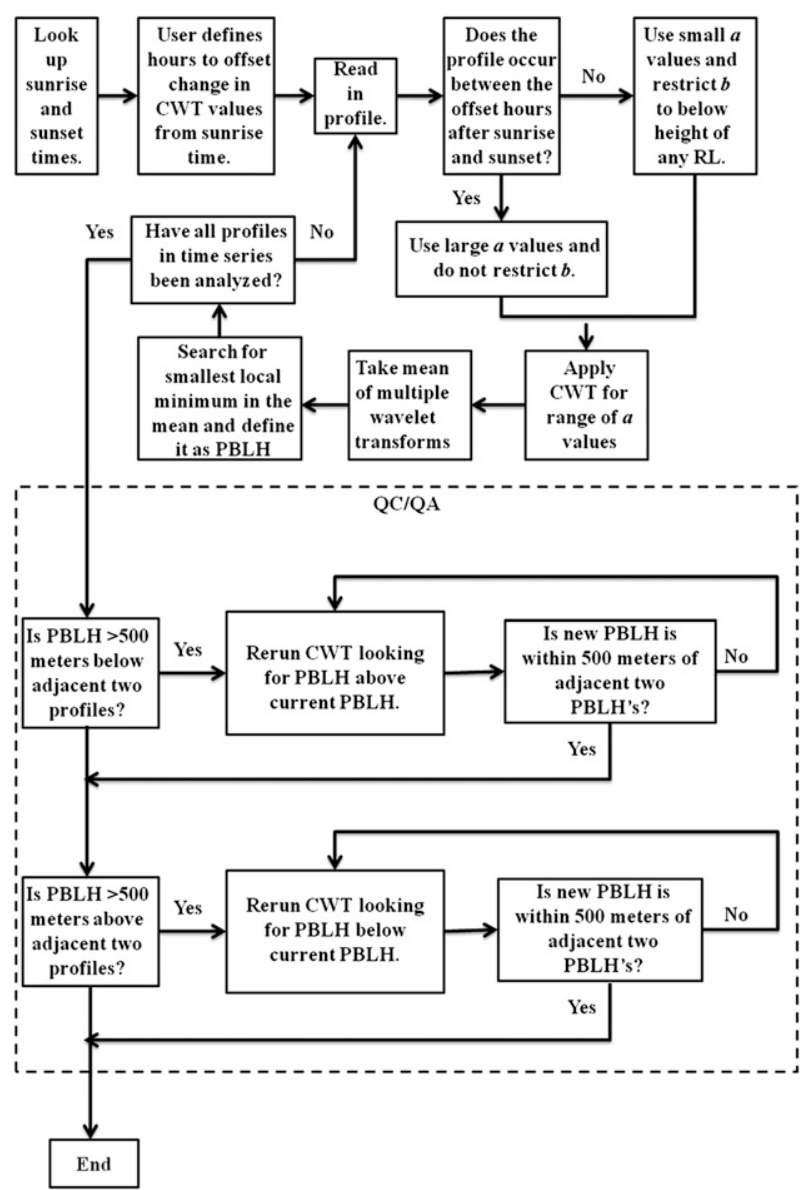

FIG. 2. Schematic representation of the PBLH detection algorithm.

the Haar function value $b$ is defined as a height parameter that goes from the first recorded height to the maximum altitude desired for the algorithm to search for PBLH. The dilation value of the Haar function $a$ is the size of the window that the algorithm is looking for a gradient. It is the most important and difficult factor to automate in this method. Figure 3 shows the CWT coefficient profiles of different dilation values for lidar attenuated backscatter and wind profiler SNR profiles for 16 July 2011. A small dilation value will produce local minimums at heights of all the gradients, small and large, in the lidar profile, such as the small gradients of a lower aerosol layer and the large gradient that occurs at the top of the PBL. This is because, at small dilations of the Haar function, the algorithm detects small gradients and noise in the profile. This can be seen in Fig. $3 \mathrm{a}$, where numerous local minimums are produced for the dilation value of $37.5 \mathrm{~m}$ as a result of small changes in the lidar profile. This can also be seen in Fig. 3b, where for the dilation value of $200 \mathrm{~m}$ for the wind profiler strong local minimums are produced, such as above and below $1.5 \mathrm{~km}$, as a result of changes in the SNR profile.

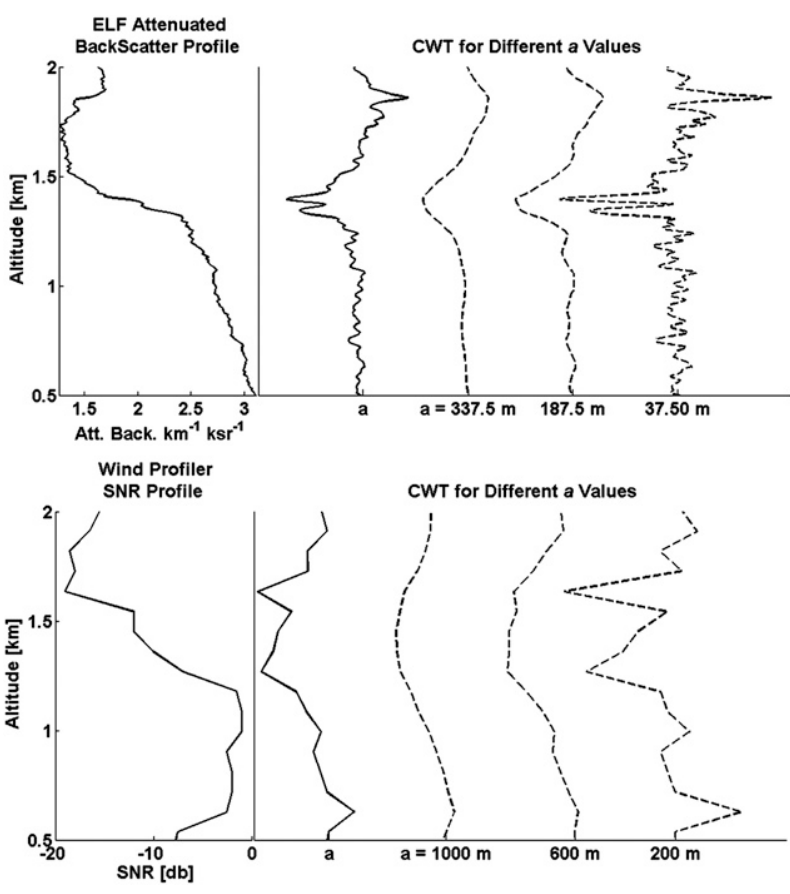

FIG. 3. ELF attenuated backscatter profile (top left) at 1452 UTC 16 Jul 2011 and (top right) the CWT coefficient profiles (dashed lines) for dilation values of $37.5,187.5$, and $337.5 \mathrm{~m}$. Wind profiler SNR profiles for the (bottom left) same day and time and (bottom right) the CWT coefficient profile (dashed lines) for dilation values of 200,600 , and $1000 \mathrm{~m}$. Minimum value of mean of the CWT profiles is selected as the PBLH.

A large dilation value will produce local minimums at the height of the larger gradients in the profile and ignore the smaller changes that occur also in the profile. The dilation values of 337.5 and $1000 \mathrm{~m}$ for the lidar and wind profiler profiles, respectively, produce local minimums at heights of the largest gradient changes in the profiles. Large dilation values reduce precision in determining the PBLH by smearing the profile. There are advantages and disadvantages of using small and large dilation values. The use of a small dilation value is advantageous during the early morning hours when a strong residual layer is present because we can search for smaller features beneath it, but disadvantageous later in the day when only the largest gradients are of interest and large dilation values are advantageous. Large dilation values are problematic during the early morning hours, when it may falsely detect the top of the residual layer, which may be the location of the largest gradient, instead of a shallow-growing PBL. Automating a precise dilation value to use on a lidar profile that highlights the gradients of interest is difficult. The algorithm applies a range of dilation values to the profile. This range of dilations is incremented in a way that, after calculating the mean of the CWT coefficients for all the dilations is 
taken, a local minimum can be automatically be searched for and found. In most cases, the local minimum with the smallest coefficient value is selected as the PBLH; however, in the early part of the day when a strong residual layer is present, the local minimum below a specific altitude $(1 \mathrm{~km}$ is hard coded in the algorithm, since prior-day PBLH's are generally greater than this) is selected.

Figure 2 shows the flowchart for the PBLH detection algorithm. It should be emphasized that this technique is designed to look for elevated convection daytime boundary layer heights. Nocturnal layers may be driven by weak mechanical turbulence, not buoyancy, and they tend to be very shallow. None of the three techniques used here has sufficient vertical resolution to see nocturnal PBLHs, and we confine ourselves to times when the PBLH has begun to rise in the morning and before it collapses in the evening. The first step is to look up sunrise and sunset times. The algorithm uses two sets of $a$ and $b$ values based on the time of day. One set of values is used before the PBL begins to grow and another set is used after it begins to grow. The user of this algorithm must define a number of hours after sunrise to offset the change in values. This number of hours (typically $2 \mathrm{~h}$ ) after the sunrise time is referred to as the start time. The start time must be defined by the user because it can change from day to day because of the variability of atmospheric conditions, which affects the growth rate of the PBL (such as high surface moisture after a storm). Once the start time has been defined, the profile is read in. If the profile occurs before the start time, then $b$ is restricted to below the top of the RL and only small $a$ values are used. If the profile occurs between the start and sunset times, then $b$ is not restricted and larger $a$ values are used. Once the $a$ and $b$ values have been defined, then the CWT is applied. The mean of the CWT is taken and is searched for the smallest local minimum, which is defined as the PBLH. After this a quality control/quality assurance (QC/QA) check (Fig. 2, boxed area) is performed to find outlier PBLHs that were incorrectly selected by the CWT. This is done by checking if the PBLH is greater than $500 \mathrm{~m}$ above or below the PBLHs of the adjacent two profiles. If this is true, then the CWT is applied continuously until the PBLH of the profile is within $500 \mathrm{~m}$ of the adjacent two profiles. If the PBLH in question is greater than $500 \mathrm{~m}$ above the PBLHs of the adjacent two profiles, then the CWT is reapplied to the profile for the same values of $a$, but the value of $b$ is set as the PBLH that was last calculated for the profile. By setting the value of $b$ to the PBLH that was last calculated for the profile, the algorithm searches for the smallest local minimum below this value. If the $\mathrm{PBLH}$ in question is greater than $500 \mathrm{~m}$ below the
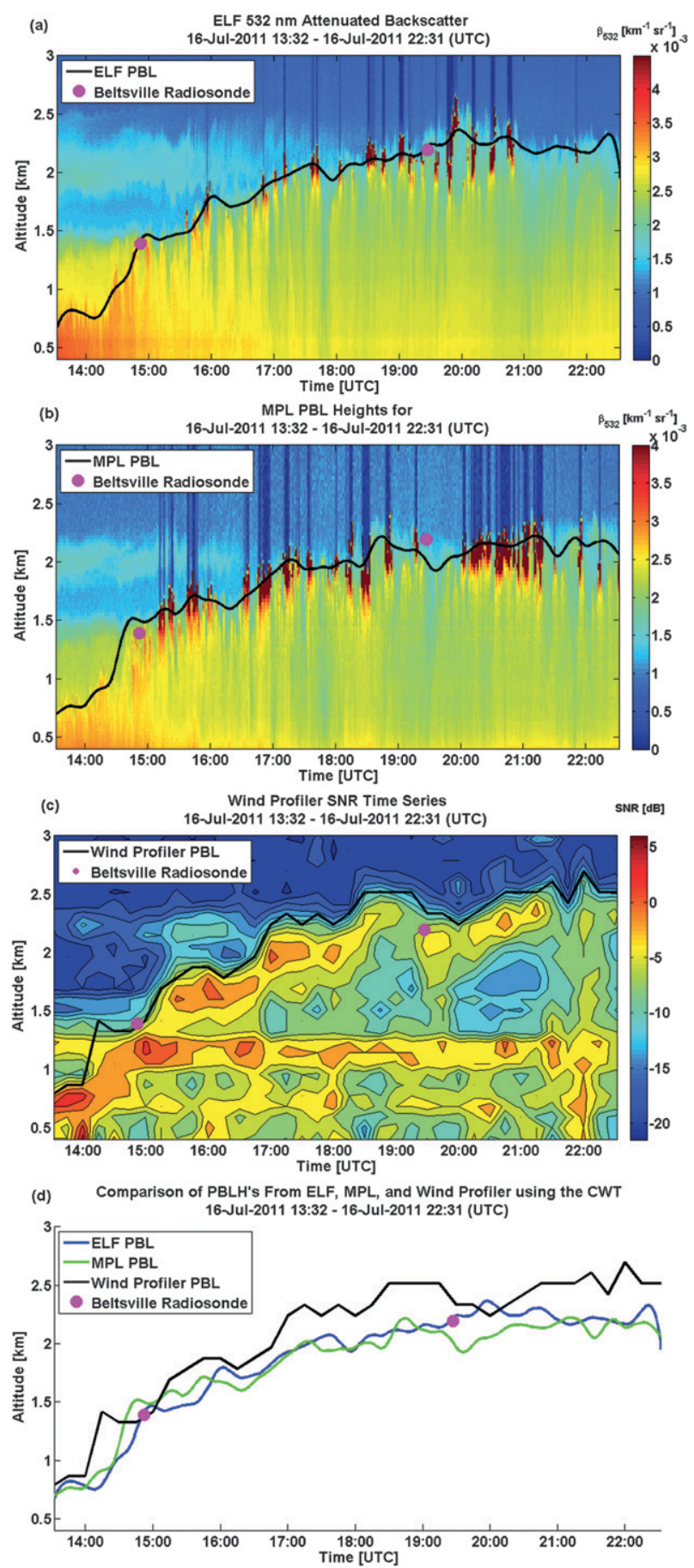

FIG. 4. (a) ELF and (b) MPL attenuated backscatter time series for 16 Jul 2011, with the PBLH derived using the CWT (black line) overlaid. (c) Wind profiler SNR time series for the same day with PBLH derived using the CWT overlaid. (d) Plot of PBLH from each of the three instruments. All images have radiosonde launches from Beltsville, MD, plotted as maroon dots. Each instrument shows a PBLH within $200 \mathrm{~m}$ of the radiosonde PBLH. All three methods follow the growth of the PBL, with the largest difference between the wind profiler PBLH and the lidars PBLH occurring at 1415 UTC. 
PBLHs of the adjacent two profiles, then the algorithm uses the same values of $a$ and searches for local minimums that occur between the PBLH of the last calculated profile and the $b$ value.

\section{Comparison of radiosonde-derived PBLHs to lidar and wind profiler CWT-derived PBLHs}

Figure 4 shows time series for ELF, MPL, and the wind profiler for 16 July 2011 overlaid on two radiosonde launches from Beltsville. Figures $4 \mathrm{a}-\mathrm{c}$ show the ELF and MPL attenuated backscatter, and wind profiler SNR time series, respectively. For each of the three instruments, the PBL starts off early in the time series at 1332 UTC (0932 local time for ELF, which is $4 \mathrm{~h}$ behind UTC in summer) at approximately $600 \mathrm{~m}$, with a strong RL layer above it that extends up to approximately $1.50 \mathrm{~km}$. The CWT is successfully able to distinguish the PBL from the RL for each of the three instruments. The PBL is observed by each of the instruments beginning to grow from the NBL into the ML just after 1400 UTC. The CWT follows the rise of the PBL in each of the three instruments. The PBLH determined by the radiosonde, ELF, MPL, and wind profiler for this radiosonde launch are listed in Table 1. The first radiosonde of the day was launched at 1448 UTC and by 1452 UTC it had reached the PBLH. For this launch, the wind profiler showed the best agreement with the PBLH from the radiosonde. The PBL rises at both locations steadily from approximately 1.50 to $2.20 \mathrm{~km}$ between this radiosonde launch and the second radiosonde launch at 1921 UTC, which reached the PBLH at 1927 UTC. ELF showed the best agreement with the radiosonde PBLH for the second launch. In Fig. 4d, the PBLHs for all the instruments are shown. The PBLHs for ELF and the MPL closely follow each other throughout the day, reporting similar PBLHs between approximately 1630 and 1930 UTC. The largest disagreements between the two were $420 \mathrm{~m}$ just after 1430 UTC and $345 \mathrm{~m}$ just after 1930 UTC. The MPL and the ELF lidars agree well in PBLH with a regression of $\operatorname{PBLH}(\mathrm{MPL})=(0.91 \pm 0.00)$ PBLH $(\mathrm{ELF})$ with correlation coefficient $R^{2}=0.85$. The bias between the two instruments over all measurements is just $20 \mathrm{~m}$. For the
TABLE 1. PBLHs from the different instruments for 16 Jul 2011 at times when radiosondes were launched.

\begin{tabular}{ccccc}
\hline \hline $\begin{array}{c}\text { Radiosonde } \\
\text { launch } \\
(\text { UTC })\end{array}$ & $\begin{array}{c}\text { Radiosonde } \\
\text { PBLH } \\
(\mathrm{km})\end{array}$ & $\begin{array}{c}\text { ELF } \\
\text { PBLH } \\
(\mathrm{km})\end{array}$ & $\begin{array}{c}\text { MPL } \\
\text { PBLH } \\
(\mathrm{km})\end{array}$ & $\begin{array}{c}\text { Wind } \\
\text { profiler } \\
\text { PBLH }(\mathrm{km})\end{array}$ \\
\hline 1452 & 1.39 & 1.40 & 1.50 & 1.41 \\
1927 & 2.19 & 2.23 & 2.03 & 2.3 \\
\hline
\end{tabular}

most part, the differences between the wind profiler and the lidars are within 400-500 m.

Table 2 shows the bias of the wind profiler PBLHs compared to PBLHs from radiosondes launched during DISCOVER-AQ. One to two radiosondes per day were launched over the course of 14 days in July. The wind profiler has a bias of $140 \mathrm{~m}$ with a standard deviation of $180 \mathrm{~m}$. ELF and MPL lidars have a bias of 30 and $-20 \mathrm{~m}$ with a standard deviation of 230 and $180 \mathrm{~m}$, respectively. The wind profiler has a much larger bias than the lidars.

Figure 5 shows the linear regression comparisons of PBLHs between the instruments and the radiosonde launches to validate the accuracy of the CWT applied to the instruments. The results are summarized in Table 2. The wind profiler showed the highest agreement with the radiosondes $\left(R^{2}=0.94\right.$, slope $\left.=0.98\right)$, but it also had the highest bias of $140 \mathrm{~m}$ above the radiosonde PBLHs. The MPL showed the second highest agreement $\left(R^{2}=\right.$ 0.92 , slope $=0.83$ ) with a bias of $20 \mathrm{~m}$ below the radiosonde PBLHs. While not far below the other two instruments, ELF had the lowest agreement $\left(R^{2}=0.89\right.$, slope $=0.82)$ with a bias of $20 \mathrm{~m}$ above the radiosonde PBLHs. Comparisons between all of the instruments were done in Fig. 6 with the results summarized in Table 3. The linear regression comparison between PBLHs from the MPL and ELF was done over the course of 22 days from 11 to 29 July 2011. The lidars show good agreement with each other $\left(R^{2}=0.85\right.$, slope $\left.=0.91\right)$, with ELF having a bias of $20 \mathrm{~m}$ above the MPL. This shows there is little to no variability between the two suburban sites that are $29 \mathrm{~km}$ apart. Figures $6 \mathrm{~b}$ and $6 \mathrm{c}$ show the linear regression comparisons between the PBLHs calculated from the wind profiler and PBLHs calculated from ELF and the MPL, respectively. PBLHs from the wind profiler and ELF were compared over the course of 14 days

TABLE 2. Comparisons between PBLH derived from the radiosondes launched during the DISCOVER-AQ campaign and the PBLH determination techniques for each instrument.

\begin{tabular}{lcccr}
\hline \hline Instrument & $N$ (No. of points) & $R^{2}$ & $y=m x+b$ & Bias $(\mathrm{km})$ \\
\hline ELF & 23 & 0.89 & $(0.82 \pm 0.06) x+(0.28 \pm 0.10)$ & $0.03 \pm 0.23$ \\
MPL & 17 & 0.92 & $(0.83 \pm 0.06) x+(0.21 \pm 0.09)$ & $-0.02 \pm 0.18$ \\
Wind profiler & 25 & 0.94 & $(0.98 \pm 0.05) x+(0.17 \pm 0.08)$ & $0.14 \pm 0.18$ \\
\hline
\end{tabular}


(a)

ELF vs. Radiosonde

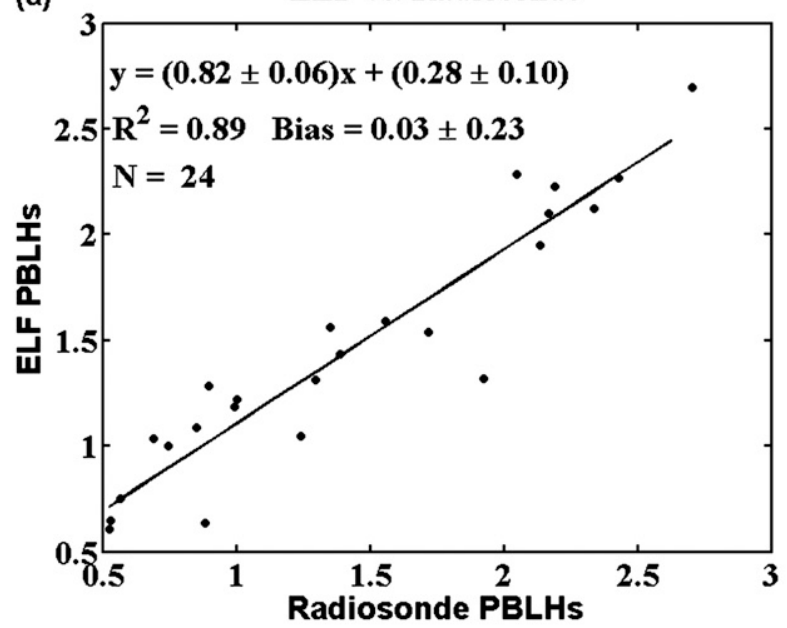

(b)

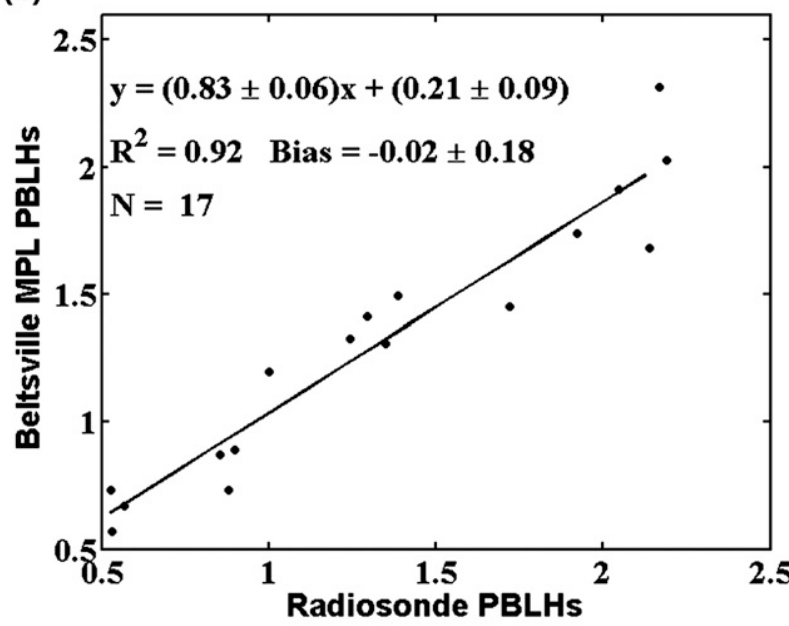

(c)

Wind Profiler vs. Radiosonde

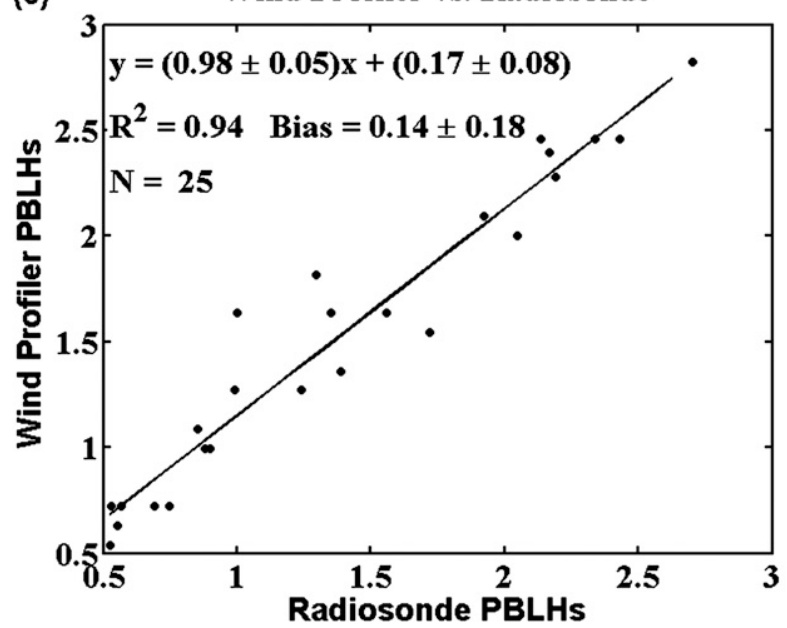

FIG. 5. Linear regressions comparing PBLH's from radiosondes to (a) ELF, (b) MPL, and (c) wind profiler. The slope, intercept, $R^{2}$, and number of data points compared are listed.
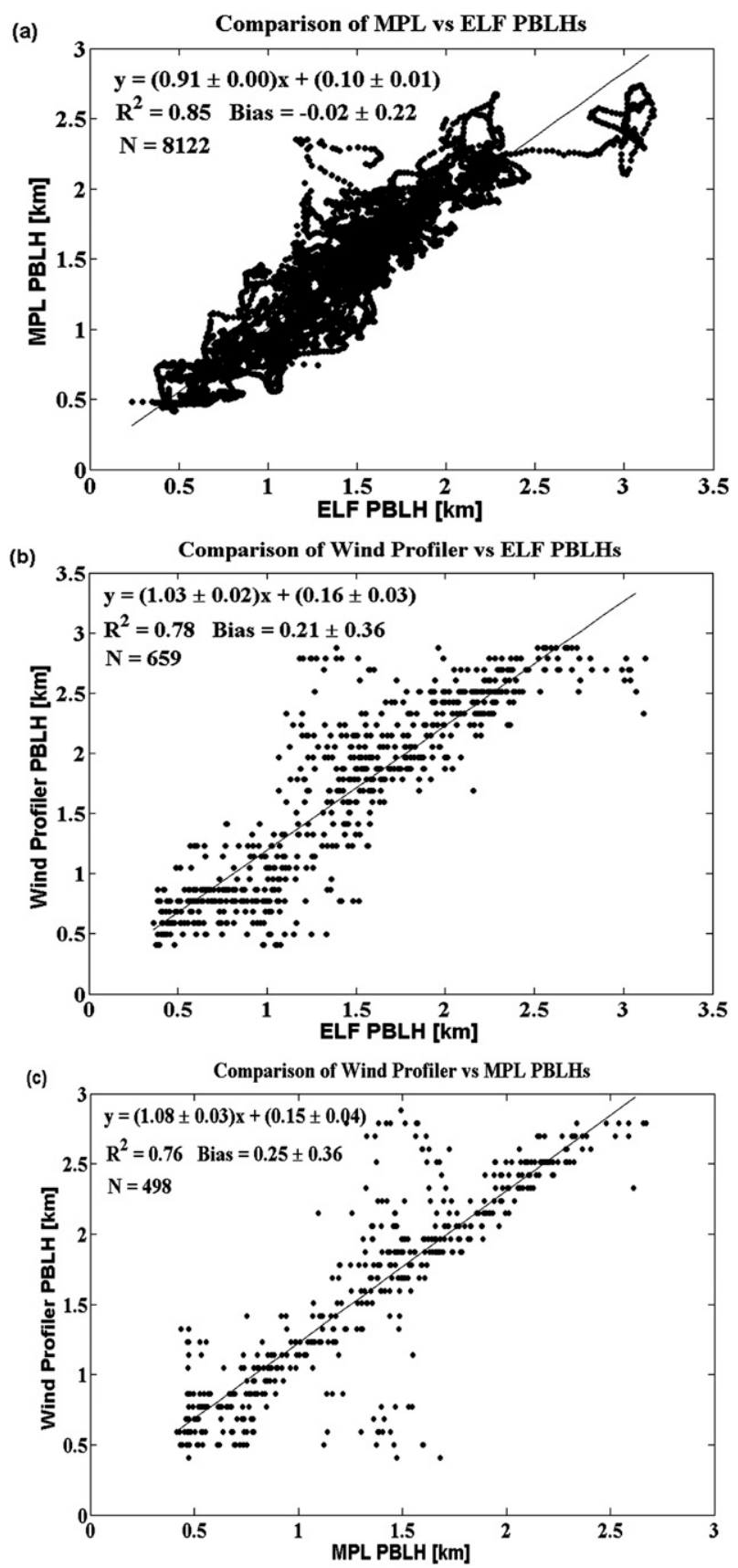

FIG. 6. Linear regressions comparing PBLH (a) from MPL to ELF, (b) from wind profiler to ELF, and (c) from wind profiler to the MPL. The slope, intercept, $R^{2}$, and number of data points compared are shown.

in July 2011, while PBLHs from the wind profiler and the MPL were compared over the course of 10 days from 11 to 29 July 2011. The PBLHs from the wind profiler showed the best agreement with PBLHs from the MPL $\left(R^{2}=0.76\right.$, slope $\left.=1.08\right)$ with a bias of $250 \mathrm{~m}$ above the MPL PBLHs. The wind profiler had a lower agreement $\left(R^{2}=0.74\right.$, slope $\left.=1.01\right)$ and a bias of $150 \mathrm{~m}$ above the 
TABLE 3. Comparisons between PBLH derived for MPL vs ELF, wind profiler vs ELF, and wind profiler vs MPL.

\begin{tabular}{lcccr}
\hline \hline \multicolumn{1}{c}{ Instruments } & $N$ (No. of points) & $R^{2}$ & $y=m x+b$ & Bias $(\mathrm{km})$ \\
\hline MPL vs ELF & 8106 & 0.85 & $(0.91 \pm 0.00) x+(0.10 \pm 0.01)$ & $-0.02 \pm 0.22$ \\
Wind profiler vs ELF & 659 & 0.74 & $(1.01 \pm 0.02) x+(0.19 \pm 0.04)$ & $0.19 \pm 0.39$ \\
Wind profiler vs MPL & 498 & 0.76 & $(1.08 \pm 0.03) x+(0.14 \pm 0.04)$ & $0.25 \pm 0.35$ \\
\hline
\end{tabular}

PBLHs from ELF. From this, the wind profiler shows a bias of $150-250 \mathrm{~m}$ above the lidars and radiosondes. This could be partially due to the vertical resolution of the wind profiler $(100 \mathrm{~m})$. Another factor, however, that remains unresolved is whether the ground clutter near HUBRC might lead to a systematic first bin offset that affects the wind profiler and not the lidars. It is concluded that the application of the CWT on the wind profiler and the lidars has been demonstrated by the high level of agreement between the radiosondes PBLHs and the wind profiler and lidar PBLHs.

\section{Conclusions}

The application of the CWT on lidar attenuated backscatter and wind profiler SNR profiles to determine the height of the PBL has been carried out. The application of the CWT on lidar profiles for the purpose of detecting PBL heights from lidar has been examined in previous studies (Brooks 2003; Cohn and Angevine 2000); however, the application of the CWT on wind profiler SNR profiles has not. Similar to lidar profiles, which observe a sharp gradient in attenuated backscatter at the top of the PBL, wind profiler SNR profiles observe a sharp gradient in SNR values at the top of the boundary layer as a result of the sharp decrease in humidity at the top of the PBL.

The reviewers of this paper raised the question that if Angevine et al.'s (1994) assertion that a local maximum in the wind profiler SNR, due to maxima in $C_{n}^{2}$, identifies the PBLH, why not use a peak detection algorithm or a symmetric wavelet such as the "Mexican hat" wavelet? The supplemental material shows that both a peak detection algorithm and Mexican hat wavelet perform poorer than the Haar CWT (supplemental material is available at the Journals Online website: http://dx.doi. org/10.1175/JTECH-D-12-00116.s1). Reflection on the physics of the problem explains why. Ottersten (1969) correctly points out that $C_{n}^{2}$ reflects index of refraction changes from humidity gradients. Angevine et al. also correctly point out that these gradients are large at the top of the PBL. But humidity gradients can be embedded within the PBL when convective, upwelling wet air is bounded by downwelling dry air. These gradients can also be large. Use of a peak detection algorithm or a symmetric wavelet can get fooled by such embedded structures within the PBL and give an oscillatory detection of the PBLH. The Haar wavelet, however, looks for features where the SNR decreases from the PBL to the free troposphere aloft (an asymmetric filter).

The Haar CWT can be used on both types of instruments to detect PBLHs. The dilation value of the CWT is the most important and difficult value to automate. Small dilation values reveal gradients in the SNR and attenuated backscatter profiles that occur at smaller height intervals, while larger dilations reveal gradients in the profiles that occur over larger height intervals. The CWT is applied on the short temporal resolution (1 and $15 \mathrm{~min}$ ) profiles for a range of dilation value. Local minima in the wavelet covariances are produced at the heights where gradient decreases occur in the profiles. The sharper the gradient decreases, the smaller the local minimum. The mean of the wavelet covariances is taken and then searched for the altitude in which the smallest local minimum occurs, typically at the height of the PBL.

Radiosondes launched from Beltsville, Maryland, during DISCOVER-AQ in July 2011 and PBLHs detected by application of the CWT on attenuated backscatter profiles from two lidars, MPL at Beltsville and ELF at UMBC, were used to validate the application of the CWT to wind profiler data. Comparisons with PBLHs detected from radiosondes show the PBLHs detected from the wind profiler using the CWT had the highest agreement compared to the MPL and ELF. This shows that the CWT can be successfully applied to wind profiler SNR profiles to detect PBLHs. With the national radiosonde network in decline and insufficient for global monitoring, there is a growing need for a "network of networks" of new and existing radiosonde launch sites, wind profilers, and lidar to address the current inadequacies in determining the PBLH (NRC 2000, 2009). The results shown here demonstrate the ability of lidars and wind profiler to accurately detect PBLH's through the use of the CWT.

Acknowledgments. The authors gratefully acknowledge support for this study, which was provided by the Maryland Department of the Environment (Contract U00P7201032), NOAA-CREST CCNY Foundation CREST Grant (Contract NA11SEC481004), and NASA Grant (DISCOVER-AQ NNX10AR38G). We thank Laura Warren and Dr. Everette Joseph for providing 
wind profiler and sounding data, respectively. We gratefully acknowledge Sigma Space for providing MPL systems during the 2011 NASA DISCOVER-AQ campaign. Jaime Compton would like to acknowledge DeTect Inc. for its support during the development of the PBLH detection algorithm for their Raptor DBS-BL LAP-3000 Upgrade radar wind profiler.

\section{REFERENCES}

Angevine, W. M., A. B. White, and S. K. Avery, 1994: Boundary layer depth and entrainment zone characterization with a boundary-layer profiler. Bound.-Layer Meteor., 68, 375385.

Baars, H., A. Ansmann, R. Engelmann, and D. Althausen, 2008: Continuous monitoring of the planetary-boundary-layer depth with lidar. Atmos. Chem. Phys., 8, 7281-7296.

Bell, M. L., A. McDermott, S. L. Zeger, J. M. Sarnet, and F. Dominici, 2004: Ozone and short-term mortality in 95 US urban communities, 1987-2000. J. Amer. Med. Assoc., 292, 2372-2378.

Brooks, I. M., 2003: Finding boundary layer top: Application of a wavelet covariance transform to lidar backscatter profiles. J. Atmos. Oceanic Technol., 20, 1092-1105.

Cohn, S., and W. Angevine, 2000: Boundary layer height and entrianment zone thickness measured by lidars and windprofiling radars. J. Appl. Meteor., 39, 1233-1247.

Comer, J. P., 2003: UMBC Elastic Backscatter Lidar Facility (ELF): Subvisible cirrus cloud and aerosol measurements during ABOVE 2002. M.S. thesis, Dept. of Physics, University of Maryland, Baltimore County, $128 \mathrm{pp}$.

Davis, K. J., N. Gamage, C. R. Hagelberg, C. Kiemle, D. H. Lenschow, and P. P. Sullivan, 2000: An objective method for deriving atmospheric structure from airborne lidar observations. J. Atmos. Oceanic Technol., 17, 1455-1468.

Engel-Cox, J. A., and Coauthors, 2006: Integrating lidar and satellite optical depth with ambient monitoring for 3-dimensional particulate characterization. Atmos. Environ., 40, 8056-8067.

Gamage, N., and C. Hagelberg, 1993: Detection and analysis of microfronts and associated coherent events using localized transforms. J. Atmos. Sci., 50, 750-756.

Lippmann, M., 1991: Health effects of tropospheric ozone. Environ. Sci. Technol., 25, 1954-1962.

Melfi, S. H., J. D. Spinhirne, S.-H. Chou, and S. P. Palm, 1985: Lidar observations of vertically organized convection in the planetary boundary layer over the ocean. J. Climate Appl. Meteor., 24, 806-821.

NRC, 2000: Improving Atmospheric Temperature Monitoring Capabilities: Letter Report. National Academy Press, $18 \mathrm{pp}$.

- 2009: Observing Weather and Climate from the Ground Up: A Nationwide Network of Networks. National Academy Press, $250 \mathrm{pp}$

Ottersten, H., 1969: Atmospheric structure and radar backscattering in clear air. Radio Sci., 4, 1179-1193.

Seibert, P., F. Beyrich, S. Gryning, S. Joffre, A. Rasmussen, and P. Tercier, 2000: Review and intercomparison of operational methods for the determination of the mixing height. Atmos. Environ., 34, 1001-1027.

Steyn, D. G., M. Baldi, and R. M. Hoff, 1999: The detection of mixed layer depth and entrainment zone thickness from lidar backscatter profiles. J. Atmos. Oceanic Technol., 16, 953959.

Stull, R. B., 1988: An Introduction to Boundary Layer Meteorology. Kluwer Academic Publishers, 666 pp.

WHO, 2012: Health risks of particulate matter from long-range transboundary air pollution. Joint WHO/Convention Task Force on the Health Aspects of Air Pollution, WHO Rep. E88189, 99 pp. 